\title{
Ovarian Clear Cell Cystadenocarcinoma
}

National Cancer Institute

\section{Source}

National Cancer Institute. Ovarian Clear Cell Cystadenocarcinoma. NCI Thesaurus. Code C7980.

A malignant glandular epithelial neoplasm arising from the ovary. It is characterized by the presence of clear and hobnail cells and cystic structures. 